\title{
Some Similarity Measures for Triangular Fuzzy Number and Their Applications in Multiple Criteria Group Decision-Making
}

\author{
Liyuan Zhang, ${ }^{1,2}$ Xuanhua $\mathrm{Xu},{ }^{1}$ and Li Tao ${ }^{3}$ \\ ${ }^{1}$ School of Business, Central South University, Changsha, Hunan 410083, China \\ ${ }^{2}$ School of Business, Shandong University of Technology, Zibo, Shandong 255049, China \\ ${ }^{3}$ School of Science, Shandong University of Technology, Zibo, Shandong 255049, China \\ Correspondence should be addressed to Liyuan Zhang; zhangliyuancsu@163.com
}

Received 19 September 2012; Accepted 11 March 2013

Academic Editor: Saeid Abbasbandy

Copyright (C) 2013 Liyuan Zhang et al. This is an open access article distributed under the Creative Commons Attribution License, which permits unrestricted use, distribution, and reproduction in any medium, provided the original work is properly cited.

\begin{abstract}
We propose some similarity measures between two triangular fuzzy numbers (TFNs) based on the vector similarity measures in vector space, which can be used to aggregate the decision information with TFNs. A methodology for multiple criteria group decision-making (MCGDM) problems with triangular fuzzy information is proposed; the criteria values take the form of linguistic values, which can be converts to TFNs. According to the weighted similarity measures between each alternative and ideal alternative, it is easy to rank alternatives and select the most desirable alternative. Finally, we apply the proposed methods to an illustrative example of MCGDM; the numerical results show that our method is effective and practical. For comparison, we also apply our similarity measures method to solve the fuzzy decision-making problem in Wei (2011); our method has simpler computation and gets the same results more rapidly than the FLOWHM method.
\end{abstract}

\section{Introduction}

Fuzzy multiple criteria decision-making (FMCDM) is the process of ranking the feasible alternatives and selecting the best one by considering multiple criteria, in which the alternatives and criteria values are carried by fuzzy sets [1], intuitionistic fuzzy set $[2,3]$, triangular fuzzy number [4], triangular intuitionistic fuzzy number [5], interval-valued triangular fuzzy number [6], trapezoidal fuzzy number [7, 8], intuitionistic trapezoidal fuzzy number [9], internalvalued trapezoidal fuzzy number [10], and so on. As an important part of the modern decision science, some related methods have been successfully applied to fuzzy decisionmaking problems, for example, ordered weighted aggregation operators [11], weighted geometric aggregation operators [10], TOPSIS method [12-14], analytic hierarchy process method [15], grey relational analysis method [16, 17], and similarity measures $[3,18]$, and so forth. Over the last decades, many studies have been done on the concepts of similarity measures between two intuitionistic fuzzy sets. On the one hand, the similarity measures were defined based on distance models, such as the Hamming distance similarity method [19], the Hausdorff distance similarity measure [20], and the Euclidean distance similarity measure [3]. On the other hand, the intuitionistic fuzzy set was seen as a vector containing some elements, by using the vector similarity measures to define the similarity measures between two intuitionistic fuzzy sets, for example, the cosine similarity measures [21] and so forth. However, as an effective method and a wide range of application in various fields, very few researchers worked on similarity measures between two triangular fuzzy numbers and applied them to triangular fuzzy multiple criteria group decision-making.

Because the triangular fuzzy number (TFN) is intuitive, easy to use, computationally simple, and useful in promoting representation and information processing in a fuzzy environment, it was usefully applied to solve FMCDM problems, in which the criteria values are carried by the TFNs. Many methodologys have been proposed to solve FMCDM with TFNs in the literature; Fu [22] presented a fuzzy optimization method based on the concept of ideal and anti-ideal points to solve FMCDM problems with TFNs. A case study of 


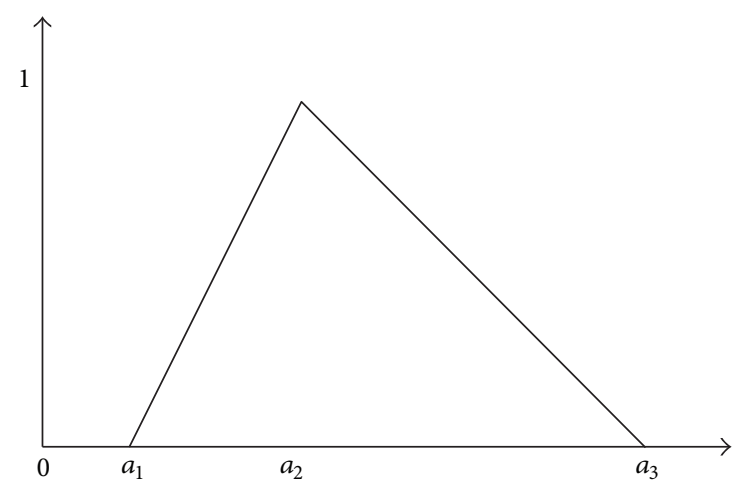

FIgURE 1: Membership functions of TFNs.

reservoir flood control operation was given to demonstrate the proposed method's effectiveness. Wei [23] proposed fuzzy induced ordered weighted harmonic mean operator to solve fuzzy group decision-making problems. In [24], Wei et al. investigated the multiple attribute decision-making problems with triangular fuzzy information and developed an generalized triangular fuzzy correlated averaging operator; numerical results showed the method was applicable. The above literatures mainly focus on the group decision-making with unknown information on criterion weight and on group member weight. However, in some actual decisionmaking process, the information about criterion weight and on group member weight are important. Therefore, with the defects of the methods in the literatures, we develop some similarity measures to solve FMCGDM problems with known information on criterion weight and on group member weight. In order to do so, the rest of this paper is set out as follows. In the next section, we introduce some basic concepts and operational laws of TFNs; three similarity measures between two TFNs are also defined. In Section 3, we present a triangular FMCGDM method based on similarity measures. Section 4 gives two illustrated examples to verify the developed approach; all numerical results show that our method is feasible and applicable. The paper ends with conclusion in Section 5.

\section{Preliminaries}

We consider the following well-know description of a triangular fuzzy number $\alpha$.

Definition 1 (see [25]). A triangular fuzzy number $\alpha$ can be defined by a triplet $\left(a_{1}, a_{2}\right.$, and $\left.a_{3}\right)$. The membership function $\mu_{\alpha}(x)$ is

$$
\mu_{\alpha}(x)= \begin{cases}0, & x<a_{1}, \\ \frac{x-a_{1}}{a_{2}-a_{1}}, & a_{1} \leq x \leq a_{2}, \\ \frac{x-a_{3}}{a_{2}-a_{3}}, & a_{2} \leq x \leq a_{3}, \\ 0, & a_{3}<x,\end{cases}
$$

where $0 \leq a_{1} \leq a_{2} \leq a_{3} \leq 1, a_{1}$ and $a_{3}$ stand for the lower and upper values of the support of $\alpha$, respectively, and $a_{2}$ stands for the modal values. The membership functions can be seen in Figure 1.

Let $\alpha=\left(a_{1}, a_{2}, a_{3}\right)$ and $\beta=\left(b_{1}, b_{2}, b_{3}\right)$ be two TFNs and $\gamma$ a positive scalar number; the basic operational laws related to TFNs are shown as

$$
\begin{gathered}
\alpha \oplus \beta=\left(a_{1}+b_{1}, a_{2}+b_{2}, a_{3}+b_{3}\right), \\
\alpha \otimes \beta=\left(a_{1} b_{1}, a_{2} b_{2}, a_{3} b_{3}\right), \\
\gamma \otimes \alpha=\left(\gamma a_{1}, \gamma a_{2}, \gamma a_{3}\right), \\
\frac{1}{\alpha}=\left(\frac{1}{a_{1}}, \frac{1}{a_{2}}, \frac{1}{a_{3}}\right) .
\end{gathered}
$$

In the vector space, there are some similarity measures between two vectors, which successfully apply to various fields, such as pattern recognition, description and classification of complex structured objects, faculty recruitment, and fuzzy assignment problems. In this section, we introduce three important vector similarity measures.

Let $X=\left(x_{1}, x_{2}, \ldots, x_{n}\right)$ and $Y=\left(y_{1}, y_{2}, \ldots, y_{n}\right)$ be two vectors of length $n$, where all the coordinates are positive; three important similarity measures are defined as

$$
\begin{aligned}
J(Y, Z) & =\frac{X Y}{\|X\|_{2}^{2}+\|Y\|_{2}^{2}-X Y} \\
& =\frac{\sum_{i=1}^{n} x_{i} y_{i}}{\sum_{i=1}^{n} x_{i}^{2}+\sum_{i=1}^{n} y_{i}^{2}-\sum_{i=1}^{n} x_{i} y_{i}}, \\
E(Y, Z) & =\frac{2 X Y}{\|X\|_{2}^{2}+\|Y\|_{2}^{2}}=\frac{2 \sum_{i=1}^{n} x_{i} y_{i}}{\sum_{i=1}^{n} x_{i}^{2}+\sum_{i=1}^{n} y_{i}^{2}}, \\
C(Y, Z) & =\frac{X Y}{\|X\|_{2}\|Y\|_{2}}=\frac{\sum_{i=1}^{n} x_{i} y_{i}}{\sqrt{\sum_{i=1}^{n} x_{i}^{2}} \sqrt{\sum_{i=1}^{n} y_{i}^{2}}} .
\end{aligned}
$$

The three parameters $a_{i}(i=1,2$, and 3) in TFN $\alpha=$ $\left(a_{1}, a_{2}\right.$, and $\left.a_{3}\right)$ can be considered as one vector representation with three elements. Based on the extension of the similarity measures in vector space, the similarity measures between two TFNs are shown in Definition 2.

Definition 2. Let $\alpha=\left(a_{1}, a_{2}, a_{3}\right)$ and $\beta=\left(b_{1}, b_{2}, b_{3}\right)$ be two TFNs, where $0 \leq a_{1} \leq a_{2} \leq a_{3} \leq 1,0 \leq b_{1} \leq b_{2} \leq b_{3} \leq 1$; the similarity measures between two TFNs can be defined as follows:

$$
\begin{gathered}
S^{J}(\alpha, \beta)=\frac{\sum_{i=1}^{3} a_{i} b_{i}}{\sum_{i=1}^{3} a_{i}^{2}+\sum_{i=1}^{3} b_{i}^{2}-\sum_{i=1}^{3} a_{i} b_{i}}, \\
S^{E}(\alpha, \beta)=\frac{2 \sum_{i=1}^{3} a_{i} b_{i}}{\sum_{i=1}^{3} a_{i}^{2}+\sum_{i=1}^{3} b_{i}^{2}}, \\
S^{C}(\alpha, \beta)=\frac{\sum_{i=1}^{3} a_{i} b_{i}}{\sqrt{\sum_{i=1}^{3} a_{i}^{2}} \sqrt{\sum_{i=1}^{3} b_{i}^{2}}} .
\end{gathered}
$$


The three similarity measures between two TFNs in Definition 2 satisfies the following properties, as in Theorem 3.

Theorem 3. The similarity satisfies the following properties:

(1) $0 \leq S(\alpha, \beta) \leq 1$,

(2) $S(\alpha, \beta)=S(\beta, \alpha)$,

(3) if $\alpha=\beta$, that is, $a_{i}=b_{i}, i=1,2$, and 3. $S(\alpha, \beta)=$ 1.

Proof. Firstly, we prove $S^{J}(\alpha, \beta)$ satisfies the above properties, as follows.

(P1) It is obvious that $S^{J}(\alpha, \beta) \geq 0$. Thus, we only need to prove $S^{J}(\alpha, \beta) \leq 1$.

By using the basic mathematical equations,

$$
2 a_{i} b_{i} \leq a_{i}^{2}+b_{i}^{2}
$$

we get

$$
\sum_{i=1}^{3} a_{i}^{2}+\sum_{i=1}^{3} b_{i}^{2}-\sum_{i=1}^{3} a_{i} b_{i} \geq 2 a_{i} b_{i}-a_{i} b_{i} \geq a_{i} b_{i} .
$$

Taking (8) in (4), we get $S^{J}(\alpha, \beta) \leq 1$.

(P2) It is obvious that the equation is true.

(P3) When $\alpha=\beta$, that is, $a_{i}=b_{i}$, for $i=1,2$, and 3 ,

$$
S^{J}(\alpha, \beta) \frac{\sum_{i=1}^{3} a_{i} a_{i}}{\sum_{i=1}^{3} a_{i}^{2}+\sum_{i=1}^{3} a_{i}^{2}-\sum_{i=1}^{3} a_{i} a_{i}}=1 .
$$

In the same way, we can prove $S^{E}(\alpha, \beta)$ and $S^{C}(\alpha, \beta)$ satisfy the properties in Theorem 3 .

Therefore, we have finished the proofs.

\section{Triangular Fuzzy Multiple Criteria Group Decision-Making Method Based on Similarity Measures}

In this section, we present a handling method for TFNs multiple criteria group decision-making problems.

Let $C=\left\{C_{1}, C_{2}, \ldots, C_{n}\right\}$ be a set of criteria and $A=$ $\left\{A_{1}, A_{2}, \ldots, A_{m}\right\}$ a set of alternatives. Suppose we invite $p$ experts to make the judgement, and let $G=\left\{G_{1}, G_{2}, \ldots, G_{p}\right\}$ be a set of experts. They are expected to give the linguistic value of TFNs (see Table 1). The vectors of alternative $A_{i}$ given by the expert $G_{k}$ are represented by following TFNs:

$$
\begin{aligned}
v_{i}^{k}=\{ & \left\langle C_{1},\left(a_{i 11}^{k}, a_{i 12}^{k}, a_{i 13}^{k}\right)\right\rangle, \\
& \left\langle C_{2},\left(a_{i 21}^{k}, a_{i 22}^{k}, a_{i 23}^{k}\right)\right\rangle, \ldots, \\
& \left.\left\langle C_{n},\left(a_{i n 1}^{k}, a_{i n 2}^{k}, a_{i n 3}^{k}\right)\right\rangle\right\},
\end{aligned}
$$

for $i=1,2, \ldots, m, k=1,2, \ldots, p$.
The weight vector of the experts is $\lambda=\left(\lambda_{1}, \lambda_{2}, \ldots, \lambda_{p}\right)$; for each alternative $A_{i}$, the group preference vector can be calculated by

$$
\begin{aligned}
V_{i}=\{ & \left\langle C_{1},\left(\sum_{k=1}^{p} \lambda_{k} a_{i 11}^{k}, \sum_{k=1}^{p} \lambda_{k} a_{i 12}^{k}, \sum_{k=1}^{p} \lambda_{k} a_{i 13}^{k}\right)\right\rangle, \\
& \left\langle C_{2},\left(\sum_{k=1}^{p} \lambda_{k} a_{i 21}^{k}, \sum_{k=1}^{p} \lambda_{k} a_{i 22}^{k}, \sum_{k=1}^{p} \lambda_{k} a_{i 23}^{k}\right)\right\rangle, \\
& \left.\ldots,\left\langle C_{n},\left(\sum_{k=1}^{p} \lambda_{k} a_{i n 1}^{k}, \sum_{k=1}^{p} \lambda_{k} a_{i n 2}^{k}, \sum_{k=1}^{p} \lambda_{k} a_{i n 3}^{k}\right)\right\rangle\right\} .
\end{aligned}
$$

For the different critera $C_{j}(j=1,2, \ldots, n)$, the weight of a criterion is a triangular fuzzy weight, which is obtained by the decision maker, as $w_{j}=\left(a_{j 1}, a_{j 2}, a_{j 3}\right)(j=1,2, \ldots, n)$. The expected weight value $w_{j}(j=1,2, \ldots, n)$ for a triangular fuzzy weight is obtained by the following equation:

$$
E V\left(w_{j}\right)=\frac{a_{j 1}+a_{j 2}+a_{j 3}}{3} .
$$

Then we normalize the expected weight value $w_{j}(j=1,2$, $\ldots, n)$ by the following formula:

$$
\widetilde{w}_{j}=\frac{E V\left(w_{j}\right)}{\sum_{i=1}^{m} E V\left(w_{j}\right)} .
$$

In FMCGDM environments, the concept of ideal point has been used to determine the best alternative in the decision set. Although the ideal alternative does not exist in real world, it does provide a useful theoretical construct to evaluate alternatives (Ye [26]).

Thus three weighted similarity measures between an alternative $A_{i}$ and the ideal alternative $A_{P}$ represented by the TFNs are defined as follows:

$$
\begin{gathered}
S^{J}\left(A_{i}, A_{P}\right)=\sum_{j=1}^{n} w_{j} \frac{\sum_{l=1}^{3} a_{i j l} b_{p j l}}{\sum_{l=1}^{3} a_{i j l}^{2}+\sum_{l=1}^{3} b_{p j l}^{2}-\sum_{l=1}^{3} a_{i j l} b_{p j l}}, \\
S^{E}\left(A_{i}, A_{P}\right)=\sum_{j=1}^{n} w_{j} \frac{2 \sum_{l=1}^{3} a_{i j l} b_{p j l}}{\sum_{l=1}^{3} a_{i j l}^{2}+\sum_{l=1}^{3} b_{p j l}^{2}}, \\
S^{C}\left(A_{i}, A_{P}\right)=\sum_{j=1}^{n} w_{j} \frac{\sum_{l=1}^{3} a_{i j l} b_{p j l}}{\sqrt{\sum_{l=1}^{3} a_{i j l}^{2}} \sqrt{\sum_{l=1}^{3} b_{p j l}^{2}}} .
\end{gathered}
$$

The decision procedure for the proposed method can be summarized as follows.

Step 1. Give the characteristic of each alternative and criteria by the linguistic values of TFNs.

Step 2. Using (11), we can obtain the group preference vector for each alternative $A_{i}(i=1,2, \ldots, m)$. 
TABLE 1: Linguistic values of TFNs.

\begin{tabular}{lc}
\hline Linguistic term & Linguistic values of TFN \\
\hline Absolutely poor (AP) & $(0.0,0.0,0.1)$ \\
Very poor (VP) & $(0.0,0.1,0.2)$ \\
Poor (P) & $(0.1,0.2,0.3)$ \\
Medium poor (MP) & $(0.3,0.4,0.5)$ \\
Medium (M) & $(0.4,0.5,0.6)$ \\
Medium good (MG) & $(0.5,0.6,0.7)$ \\
Good (G) & $(0.7,0.8,0.9)$ \\
Very good (VG) & $(0.8,0.9,1.0)$ \\
Absolutely good (AG) & $(0.9,1.0,1.0)$ \\
\hline
\end{tabular}

Step 3. Calculate the expected weight value $w_{j}$ for a criterion $C_{j}(j=1,2, \ldots, n)$ by using (12) and (13).

Step 4. From formula (14), we can get three weighted similarity measures for an alternative $A_{i}(i=1,2, \ldots, m)$.

Step 5. Rank the alternatives, and select the best one(s) in accordance with the weighted similarity measure.

\section{Illustrative Example}

4.1. Example 1: Personnel Selection. In this section, a numerical example is presented to illustrate the application of the proposed method. Suppose that the human resources department of a company desires to hire a competent. After initial screening, four candidates (i.e., alternatives) $A_{1}, A_{2}$, $A_{3}$, and $A_{4}$ remain for further evaluation. In order to select the most suitable candidate, the decision maker invite five experts, who take into account the following five criteria: (1) emotional steadiness $\left(C_{1}\right)$; (2) oral communication skill $\left(C_{2}\right)$; (3) education experience $\left(C_{3}\right)$; (4) work experience $\left(C_{4}\right) ;(5)$ personality and self-confidence $\left(C_{5}\right)$. Assume that the five experts provide his/her preference information on candidates with regard to criteria by using a linguistic variable, as listed in Table 1.

The decision procedure for the above decision-making problem is as follows.

Step 1. Five experts provide his/her preference information on candidates with linguistic terms; we convert the linguistic variables to TFNs, as depicted in Table 2.

Step 2. The weight vector of five experts is $\lambda=(0.1,0.2$, $0.3,0.2$, and 0.2 ), and using (11), we can obtain the group preference vector for each alternative $A_{i}(i=1,2,3,4)$, as follows:

$$
\begin{aligned}
V_{1}=\{ & \left\langle C_{1},(0.13,0.17,0.22)\right\rangle,\left\langle C_{2},(0.36,0.46,0.56)\right\rangle, \\
& \left\langle C_{3},(0.39,0.54,0.69)\right\rangle,\left\langle C_{4},(0.42,0.50,0.60)\right\rangle, \\
& \left.\left\langle C_{5},(0.52,0.60,0.60)\right\rangle\right\} ;
\end{aligned}
$$

$$
\begin{array}{r}
V_{2}=\left\{\left\langle C_{1},(0.22,0.27,0.32)\right\rangle,\left\langle C_{2},(0.54,0.64,0.74)\right\rangle,\right. \\
\left\langle C_{3},(0.60,0.75,0.90)\right\rangle,\left\langle C_{4},(0.44,0.54,0.62)\right\rangle, \\
\left.\left\langle C_{5},(0.18,0.26,0.26)\right\rangle\right\} ; \\
V_{3}=\left\{\left\langle C_{1},(0.18,0.23,0.27)\right\rangle,\left\langle C_{2},(0.28,0.36,0.46)\right\rangle,\right. \\
\left\langle C_{3},(0.69,0.84,0.99)\right\rangle,\left\langle C_{4},(0.16,0.24,0.34)\right\rangle, \\
\left.\left\langle C_{5},(0.48,0.58,0.58)\right\rangle\right\} ; \\
V_{4}=\left\{\left\langle C_{1},(0.21,0.26,0.31)\right\rangle,\left\langle C_{2},(0.34,0.42,0.52)\right\rangle,\right. \\
\left\langle C_{3},(0.57,0.72,0.87)\right\rangle,\left\langle C_{4},(0.28,0.38,0.48)\right\rangle, \\
\left.\left\langle C_{5},(0.46,0.56,0.56)\right\rangle\right\} .
\end{array}
$$

Step 3. The weight value of criteria $C_{i}$ is represented by the following TFNs:

$$
\begin{aligned}
& w_{1}=\left\langle C_{1},(0.3,0.4,0.5)\right\rangle, \\
& w_{2}=\left\langle C_{2},(0.5,0.6,0.7)\right\rangle, \\
& w_{3}=\left\langle C_{3},(0.1,0.2,0.3)\right\rangle, \\
& w_{4}=\left\langle C_{4},(0.9,1.0,1.0)\right\rangle, \\
& w_{5}=\left\langle C_{5},(0.4,0.5,0.7)\right\rangle .
\end{aligned}
$$

The expected weight values can be obtained by (12). Then we normalize the expected weight value by (13) and get the weight vector $W=(0.1481,0.2222,0.0741,0.3580$, and 0.1975).

Step 4. The ideal alternative is given by the decision maker as

$$
\begin{aligned}
A_{p}=\{ & \left\langle C_{1},(0.40,0.50,0.60)\right\rangle,\left\langle C_{2},(0.70,0.80,0.90)\right\rangle, \\
& \left\langle C_{3},(0.80,0.90,1.00)\right\rangle,\left\langle C_{4},(0.70,0.80,0.90)\right\rangle, \\
& \left.\left\langle C_{5},(0.50,0.60,0.70)\right\rangle\right\} .
\end{aligned}
$$

From formula (14), we can get three weighted similarity measure for an alternative $A_{i}(i=1,2, \ldots, 4)$, as listed in Table 3.

Step 5. From Table 3, we can see that all the proposed method have the same decision results. The decision results of different similarity measures demonstrate that the proposed method for FMCGDM problem is effective.

Let us discuss the different weight $W^{*}=(0.2,0.1,0.3$, 0.20 , and 0.2 ), and using the above 4 steps, we can get the decision results as follows.

In Table 4, it is easy to see that the three similarity measures get the same decision results; the best alternative 
TABLE 2: Preference values of alternative given by 5 experts for TFNs.

\begin{tabular}{|c|c|c|c|c|c|c|}
\hline$A$ & M & $C_{1}$ & $C_{2}$ & $\mathrm{C}_{3}$ & $\mathrm{C}_{4}$ & $C_{5}$ \\
\hline \multirow{5}{*}{$A_{1}$} & 1 & $(0.0,0.0,0.1)$ & $(0.4,0.5,0.6)$ & $(0.5,0.6,0.7)$ & $(0.8,0.9,1.0)$ & $(0.5,0.6,0.7)$ \\
\hline & 2 & $(0.1,0.2,0.3)$ & $(0.3,0.4,0.5)$ & $(0.4,0.5,0.6)$ & $(0.5,0.6,0.7)$ & $(0.9,1.0,1.0)$ \\
\hline & 3 & $(0.4,0.5,0.6)$ & $(0.4,0.5,0.6)$ & $(0.1,0.2,0.3)$ & $(0.3,0.4,0.5)$ & $(0.0,0.0,0.1)$ \\
\hline & 4 & $(0.3,0.4,0.5)$ & $(0.1,0.2,0.3)$ & $(0.0,0.1,0.2)$ & $(0.5,0.6,0.7)$ & $(0.7,0.8,0.9)$ \\
\hline & 5 & $(0.5,0.6,0.7)$ & $(0.7,0.8,0.9)$ & $(0.2,0.3,0.4)$ & $(0.0,0.0,0.1)$ & $(0.5,0.6,0.7)$ \\
\hline \multirow{5}{*}{$A_{2}$} & 1 & $(0.4,0.5,0.6)$ & $(0.8,0.9,1.0)$ & $(0.5,0.6,0.7)$ & $(0.4,0.5,0.6)$ & $(0.0,0.1,0.2)$ \\
\hline & 2 & $(0.1,0.2,0.3)$ & $(0.4,0.5,0.6)$ & $(0.5,0.6,0.7)$ & $(0.8,0.9,1.0)$ & $(0.1,0.2,0.3)$ \\
\hline & 3 & $(0.7,0.8,0.9)$ & $(0.5,0.6,0.7)$ & $(0.3,0.4,0.5)$ & $(0.1,0.2,0.3)$ & $(0.0,0.1,0.2)$ \\
\hline & 4 & $(0.5,0.6,0.7)$ & $(0.7,0.8,0.9)$ & $(0.3,0.4,0.5)$ & $(0.9,1.0,1.0)$ & $(0.0,0.0,0.1)$ \\
\hline & 5 & $(0.5,0.6,0.7)$ & $(0.3,0.4,0.5)$ & $(0.4,0.5,0.6)$ & $(0.0,0.1,0.2)$ & $(0.8,0.9,1.0)$ \\
\hline \multirow{5}{*}{$A_{3}$} & 1 & $(0.3,0.4,0.5)$ & $(0.1,0.2,0.3)$ & $(0.7,0.8,0.9)$ & $(0.4,0.5,0.6)$ & $(0.5,0.6,0.7)$ \\
\hline & 2 & $(0.1,0.2,0.3)$ & $(0.5,0.6,0.7)$ & $(0.3,0.4,0.5)$ & $(0.0,0.0,0.1)$ & $(0.9,1.0,1.0)$ \\
\hline & 3 & $(0.9,1.0,1.0)$ & $(0.4,0.5,0.6)$ & $(0.5,0.6,0.7)$ & $(0.0,0.1,0.2)$ & $(0.3,0.4,0.5)$ \\
\hline & 4 & $(0.1,0.2,0.3)$ & $(0.0,0.0,0.1)$ & $(0.5,0.6,0.7)$ & $(0.4,0.5,0.6)$ & $(0.0,0.1,0.2)$ \\
\hline & 5 & $(0.4,0.5,0.6)$ & $(0.4,0.5,0.6)$ & $(0.3,0.4,0.5)$ & $(0.0,0.1,0.2)$ & $(0.7,0.8,0.9)$ \\
\hline \multirow{5}{*}{$A_{4}$} & 1 & $(0.5,0.6,0.7)$ & $(0.5,0.6,0.7)$ & $(0.4,0.5,0.6)$ & $(0.3,0.4,0.5)$ & $(0.8,0.9,1.0)$ \\
\hline & 2 & $(0.4,0.5,0.6)$ & $(0.3,0.4,0.5)$ & $(0.5,0.6,0.7)$ & $(0.0,0.1,0.2)$ & $(0.4,0.5,0.6)$ \\
\hline & 3 & $(0.3,0.4,0.5)$ & $(0.3,0.4,0.5)$ & $(0.4,0.5,0.6)$ & $(0.5,0.6,0.7)$ & $(0.7,0.8,0.9)$ \\
\hline & 4 & $(0.1,0.2,0.3)$ & $(0.0,0.1,0.2)$ & $(0.5,0.6,0.7)$ & $(0.3,0.4,0.5)$ & $(0.1,0.2,0.3)$ \\
\hline & 5 & $(0.7,0.8,0.9)$ & $(0.4,0.5,0.6)$ & $(0.1,0.2,0.3)$ & $(0.3,0.4,0.5)$ & $(0.3,0.4,0.5)$ \\
\hline
\end{tabular}

TABLE 3: Decision results of three weighted similarity measures for TFNs.

\begin{tabular}{lccr}
\hline & $S^{J}$ & $S^{E}$ & $S^{C}$ \\
\hline$A_{1}$ & 0.7869 & 0.8707 & 0.9974 \\
$A_{2}$ & 0.7976 & 0.8775 & 0.9988 \\
$A_{3}$ & 0.6367 & 0.7546 & 0.9918 \\
$A_{4}$ & 0.7528 & 0.8525 & 0.9964 \\
Ranking & $A_{2}>A_{1}>A_{4}>A_{3}$ & $A_{2}>A_{1}>A_{4}>A_{3}$ & $A_{2}>A_{1}>A_{4}>A_{3}$ \\
Best & $A_{2}$ & $A_{2}$ & $A_{2}$ \\
\hline
\end{tabular}

TABLE 4: Decision results for different weights in the same numerical example.

\begin{tabular}{lccr}
\hline & $S^{J}$ & $S^{E}$ & $S^{C}$ \\
\hline$A_{1}$ & 0.7674 & 0.8561 & 0.9959 \\
$A_{2}$ & 0.8022 & 0.8793 & 0.9971 \\
$A_{3}$ & 0.7567 & 0.8381 & 0.9950 \\
$A_{4}$ & 0.8180 & 0.8928 & 0.9984 \\
Ranking & $A_{4}>A_{2}>A_{1}>A_{3}$ & $A_{4}>A_{2}>A_{1}>A_{3}$ & $A_{4}>A_{2}>A_{1}>A_{3}$ \\
Best & $A_{4}$ & $A_{4}$ & $A_{4}$ \\
\hline
\end{tabular}

is $A_{4}$. From Tables 3 and 4 , for the different weights of the criteria, the decision results are different.

4.2. Example 2: Investment Case Selection. Using the illustrative example in [23], an investment company wants to invest a sum of money in the best option. There is a panel with five possible alternatives to invest the money: $A_{1}$ is a car company; $A_{2}$ is a food company; $A_{3}$ is a computer company; $A_{4}$ is an arms company; $A_{5}$ is a TV company. The three experts $\left(G_{1}, G_{2}\right.$, and $\left.G_{3}\right)$ must take a decision according to the following four attributes: $C_{1}$ is the risk analysis; $C_{2}$ is the growth analysis; $C_{3}$ is the social-political impact analysis; $C_{4}$ is the environmental impact analysis.

Using our methods in Section 3, the decision procedure is as follows.

Step 1. The TFNs preference vector of the three experts is shown in Table 5.

Step 2. The weight vector of three experts is $\lambda=(0.2,0.5$, and 0.3 ), and using (11), we can obtain the group preference 
TABLE 5: The TFNs preference vector of the three experts.

\begin{tabular}{cccccc}
\hline$A$ & $M$ & $C_{1}$ & $C_{2}$ & $C_{3}$ & $C_{4}$ \\
\hline \multirow{2}{*}{$A_{1}$} & 1 & $(0.80,0.85,0.90)$ & $(0.72,0.76,0.80)$ & $(0.91,0.93,0.96)$ & $(0.62,0.65,0.68)$ \\
& 2 & $(0.65,0.70,0.75)$ & $(0.57,0.61,0.65)$ & $(0.76,0.78,0.81)$ & $(0.47,0.50,0.53)$ \\
& 3 & $(0.42,0.47,0.52)$ & $(0.34,0.38,0.42)$ & $(0.53,0.55,0.58)$ & $(0.24,0.27,0.30)$ \\
\hline \multirow{3}{*}{$A_{2}$} & 1 & $(0.88,0.90,0.93)$ & $(0.67,0.77,0.83)$ & $(0.60,0.67,0.70)$ & $(0.69,0.72,0.75)$ \\
& 2 & $(0.73,0.75,0.78)$ & $(0.52,0.62,0.68)$ & $(0.42,0.52,0.50)$ & $(0.54,0.57,0.60)$ \\
& 3 & $(0.50,0.52,0.55)$ & $(0.29,0.39,0.45)$ & $(0.22,0.39,0.32)$ & $(0.31,0.34,0.37)$ \\
\hline \multirow{3}{*}{$A_{3}$} & 1 & $(0.95,0.97,0.98)$ & $(0.90,0.93,0.95)$ & $(0.77,0.79,0.82)$ & $(0.93,0.95,0.96)$ \\
& 2 & $(0.80,0.82,0.83)$ & $(0.75,0.78,0.80)$ & $(0.62,0.64,0.67)$ & $(0.78,0.80,0.81)$ \\
& 3 & $(0.57,0.59,0.60)$ & $(0.52,0.55,0.57)$ & $(0.39,0.41,0.44)$ & $(0.55,0.57,0.58)$ \\
\hline \multirow{4}{*}{$A_{4}$} & 1 & $(0.82,0.85,0.88)$ & $(0.97,0.98,1.00)$ & $(0.98,0.99,1.00)$ & $(0.97,0.99,1.00)$ \\
& 2 & $(0.67,0.70,0.73)$ & $(0.82,0.83,0.85)$ & $(0.83,0.84,0.85)$ & $(0.82,0.84,0.85)$ \\
& 3 & $(0.44,0.47,0.50)$ & $(0.59,0.60,0.62)$ & $(0.60,0.61,0.62)$ & $(0.59,0.61,0.62)$ \\
\hline \multirow{2}{*}{$A_{5}$} & 1 & $(0.78,0.79,0.81)$ & $(0.78,0.79,0.81)$ & $(0.83,0.85,0.88)$ & $(0.94,0.97,0.99)$ \\
& 2 & $(0.63,0.64,0.66)$ & $(0.63,0.64,0.60)$ & $(0.68,0.70,0.76)$ & $(0.79,0.82,0.80)$ \\
& 3 & $(0.40,0.41,0.43)$ & $(0.40,0.41,0.43)$ & $(0.45,0.47,0.50)$ & $(0.56,0.59,0.61)$ \\
\hline
\end{tabular}

TABLE 6: Decision results of different methods in the same numerical example.

\begin{tabular}{lcccc}
\hline & $S^{J}$ & $S^{E}$ & $S^{C}$ & FIOWHM operator \\
\hline$A_{1}$ & 0.6486 & 0.7141 & 0.7997 & 1.3310 \\
$A_{2}$ & 0.6465 & 0.7136 & 0.7988 & 0.6690 \\
$A_{3}$ & 0.7329 & 0.7630 & 0.7998 & 2.5000 \\
$A_{4}$ & 0.7730 & 0.7859 & 0.7999 & 4.5000 \\
$A_{5}$ & 0.7624 & 0.7803 & 0.7998 & 3.5000 \\
Ranking & $A_{4}>A_{5}>A_{3}>A_{1}>A_{2}$ & $A_{4}>A_{5}>A_{3}>A_{1}>A_{2}$ & $A_{4}>A_{5}>A_{3}>A_{1}>A_{2}$ & $A_{4}>A_{5}>A_{3}>A_{1}>A_{2}$ \\
Best & $A_{4}$ & $A_{4}$ & $A_{4}$ & $A_{4}$ \\
\hline & & &
\end{tabular}

vector for each alternative $A_{i}(i=1,2,3,4,5)$ as follows:

$$
\begin{aligned}
& V_{1}=\left\{\left\langle C_{1},(0.611,0.661,0.711)\right\rangle,\left\langle C_{2},(0.531,0.571,0.611)\right\rangle,\right. \\
&\left.\left\langle C_{3},(0.721,0.741,0.771)\right\rangle,\left\langle C_{4},(0.431,0.461,0.491)\right\rangle\right\} ; \\
& V_{2}=\left\{\left\langle C_{1},(0.691,0.711,0.741)\right\rangle,\left\langle C_{2},(0.481,0.581,0.641)\right\rangle,\right. \\
&\left.\left\langle C_{3},(0.396,0.511,0.486)\right\rangle,\left\langle C_{4},(0.501,0.531,0.561)\right\rangle\right\} ; \\
& V_{3}=\left\{\left\langle C_{1},(0.761,0.781,0.791)\right\rangle,\left\langle C_{2},(0.711,0.741,0.761)\right\rangle,\right. \\
&\left.\left\langle C_{3},(0.581,0.601,0.631)\right\rangle,\left\langle C_{4},(0.741,0.761,0.771)\right\rangle\right\} ; \\
& V_{4}=\left\{\left\langle C_{1},(0.631,0.661,0.691)\right\rangle,\left\langle C_{2},(0.781,0.791,0.811)\right\rangle,\right. \\
&\left.\left\langle C_{3},(0.791,0.801,0.811)\right\rangle,\left\langle C_{4},(0.781,0.801,0.811)\right\rangle\right\} ; \\
& V_{5}=\left\{\left\langle C_{1},(0.591,0.601,0.621)\right\rangle,\left\langle C_{2},(0.591,0.601,0.591)\right\rangle,\right. \\
&
\end{aligned}
$$

Step 3. From [23], the weight value of criteria vector $C_{i}$ is $W=$ $(0.2,0.1,0.1,0.4)$.

Step 4. The ideal alternative is given by the decision maker as

$$
\begin{aligned}
A_{p}=\{ & \left\langle C_{1},(0.44,0.47,0.50)\right\rangle,\left\langle C_{2},(0.79,0.82,0.80)\right\rangle, \\
& \left.\left\langle C_{3},(0.97,0.98,1.00)\right\rangle,\left\langle C_{4},(0.83,0.85,0.88)\right\rangle\right\} .
\end{aligned}
$$

From formula (14), we can get three weighted similarity measures for an alternative $A_{i}(i=1,2, \ldots, 5)$, as listed in Table 6. For comparison, we also list the decision results of FIOWHM operator method in Table 6.

Step 5. From Table 6, we can see that all the proposed methods have the same decision results, but our method has simpler computation and gets the same results more rapidly than the FLOWHM method.

The decision results of different methods demonstrate that the proposed method for FMCGDM problem is effective.

\section{Conclusion}

The similarity measure is an effective method to solve the FMCDM problem, but it rarely applies to triangular FMCDM 
problem. In this paper, we propose three similarity measures and three weighted similarity measures between two TFNs based on the vector similarity measures. Using the similarity measures model, we establish a method for FMCGDM with known information on criterion weight and group weight, in which the alternatives and criteria are given by triangular fuzzy information. Finally, a practical example is given to select the most suitable candidate in human resource evaluation system, by the weighted similarity measures between each alternative and ideal alternative; the ranking order of all alternatives can be determined, and the best one(s) can be easily identified as well. For comparison, we also apply our method to solve the triangular FMCGDM problem in [23]. The different methods have the same decision results, which show that our proposed method in this paper is applicable and effective.

\section{Acknowledgments}

The research is supported by the National Natural Science Foundation of China (no. 71171202), the National Social Foundation of China (no. 12AZD109), and the Science Foundation for National Innovation Research Group of China (no. 70921001).

\section{References}

[1] L. A. Zadeh, "Fuzzy sets," Information and Computation, vol. 8, pp. 338-353, 1965.

[2] H. W. Liu, "New similarity measures between intuitionistic fuzzy sets and between elements," Mathematical and Computer Modelling, vol. 42, no. 1-2, pp. 61-70, 2005.

[3] Z. S. Xu, "Some similarity measures of intuitionistic fuzzy sets and their applications to multiple attribute decision making," Fuzzy Optimization and Decision Making, vol. 6, no. 2, pp. 109121, 2007.

[4] P. D. Liu and T. J. Wang, "Method for multiple attribute decision making with triangular fuzzy number and partial attribute weight information," Journal of Information and Computational Science, vol. 4, no. 3, pp. 1017-1022, 2007.

[5] D. F. Li, "A ratio ranking method of triangular intuitionistic fuzzy numbers and its application to MADM problems," Computers and Mathematics with Applications, vol. 60, no. 6, pp. 1557-1570, 2010.

[6] S. F. Zhang, S. Y. Liu, and R. H. Zhai, "An extended GRA method for MCDM with interval-valued triangular fuzzy assessments and unknown weights," Computers \& Industrial Engineering, vol. 81, pp. 1336-1341, 2011.

[7] Z. Y. Xu, S. C. Shang, W. B. Qian, and W. H. Shu, "A method for fuzzy risk analysis based on the new similarity of trapezoidal fuzzy numbers," Expert Systems with Applications, vol. 37, no. 3, pp. 1920-1927, 2010.

[8] S. Abbasbandy and T. Hajjari, "A new approach for ranking of trapezoidal fuzzy numbers," Computers and Mathematics with Applications, vol. 57, no. 3, pp. 413-419, 2009.

[9] J. Ye, "Expected value method for intuitionistic trapezoidal fuzzy multicriteria decision-making problems," Expert Systems with Applications, vol. 38, no. 9, pp. 11730-11734, 2011.
[10] P. D. Liu, "A weighted aggregation operators multi-attribute group decision-making method based on interval-valued trapezoidal fuzzy numbers," Expert Systems with Applications, vol. 38, no. 1, pp. 1053-1060, 2011.

[11] Q. W. Cao and J. Wu, "The extended COWG operators and their application to multiple attributive group decision making problems with interval numbers," Applied Mathematical Modelling, vol. 35, no. 5, pp. 2075-2086, 2011.

[12] Z. L. Yue, "An extended TOPSIS for determining weights of decision makers with interval numbers," Knowledge-Based Systems, vol. 24, no. 1, pp. 146-153, 2011.

[13] Y. M. Wang and T. M. S. Elhag, "Fuzzy TOPSIS method based on alpha level sets with an application to bridge risk assessment," Expert Systems with Applications, vol. 31, no. 2, pp. 309-319, 2006.

[14] G. R. Jahanshahloo, F. H. Lotfi, and M. Izadikhah, "An algorithmic method to extend TOPSIS for decision-making problems with interval data," Applied Mathematics and Computation, vol. 175, no. 2, pp. 1375-1384, 2006.

[15] Y. C. Tang and C. T. Chang, "Multicriteria decision-making based on goal programming and fuzzy analytic hierarchy process: an application to capital budgeting problem," KnowledgeBased Systems, vol. 26, pp. 288-293, 2012.

[16] G. W. Wei and Y. Wei, "Model of grey relational analysis for interval multiple attribute decision making with preference information on alternatives," Chinese Journal of Management Science, vol. 16, pp. 158-162, 2008.

[17] W. L. Liu and P. D. Liu, "Hybrid multiple attribute decision making method based on relative approach degree of grey relation projection," African Journal of Business Management, vol. 4, no. 17, pp. 3716-3724, 2010.

[18] E. Szmidt and J. Kacprzyk, "A new concept of a similarity measure for intuitionistic fuzzy sets and its use in group decision making," Modeling Decisions for Artificial Intelligence, vol. 3558, pp. 272-282, 2005.

[19] Z. Liang and P. Shi, "Similarity measures on intuitionistic fuzzy sets," Pattern Recognation Letters, vol. 24, pp. 2678-2693, 2003.

[20] W. L. Hung and M. S. Yang, "Similarity measures of intuitionistic fuzzy sets based on Hausdorff distance," Pattern Recognition Letters, vol. 25, no. 14, pp. 1603-1611, 2004.

[21] J. Ye, "Cosine similarity measures for intuitionistic fuzzy sets and their applications," Mathematical and Computer Modelling, vol. 53, no. 1-2, pp. 91-97, 2011.

[22] G. T. Fu, "A fuzzy optimization method for multicriteria decision making: an application to reservoir flood control operation," Expert Systems with Applications, vol. 34, no. 1, pp. 145-149, 2008.

[23] G. W. Wei, "FIOWHM operator and its application to multiple attribute group decision making," Expert Systems with Applications, vol. 38, no. 4, pp. 2984-2989, 2011.

[24] G. W. Wei, X. F. Zhao, R. Lin, and H. J. Wang, "Generalized triangular fuzzy correlated averaging operator and their application to multiple attribute decision making," Applied Mathematical Modelling, vol. 36, no. 7, pp. 2975-2982, 2012.

[25] P. J. M. van Laarhoven and W. Pedrycz, "A fuzzy extension of Saaty's priority theory," Fuzzy Sets and Systems, vol. 11, no. 3, pp. 229-241, 1983.

[26] J. Ye, "Using an improved measure function of vague sets for multicriteria fuzzy decision-making," Expert Systems with Applications, vol. 37, no. 6, pp. 4706-4709, 2010. 


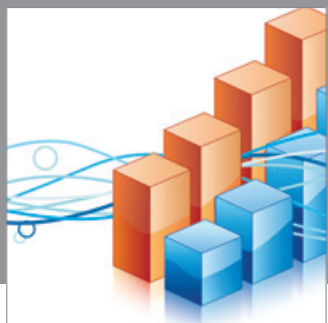

Advances in

Operations Research

mansans

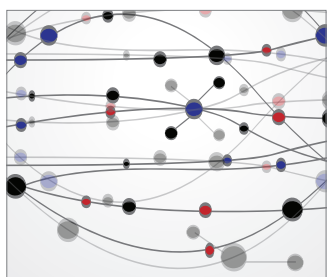

The Scientific World Journal
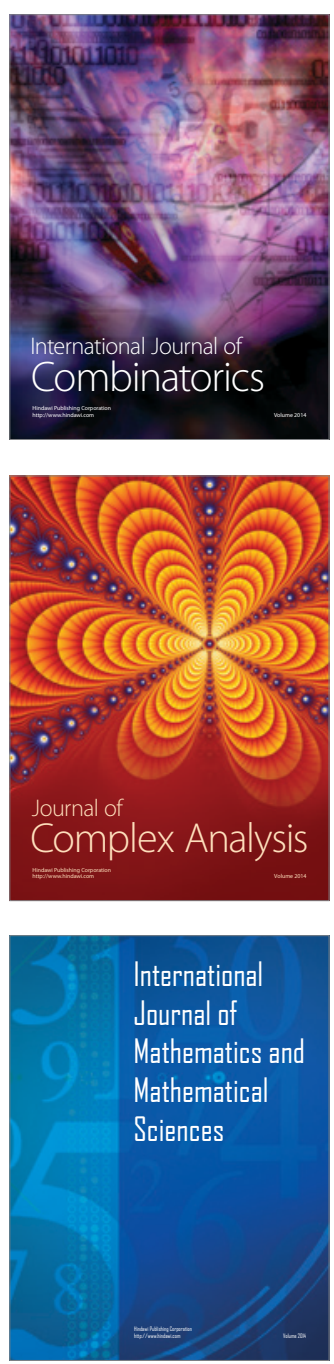
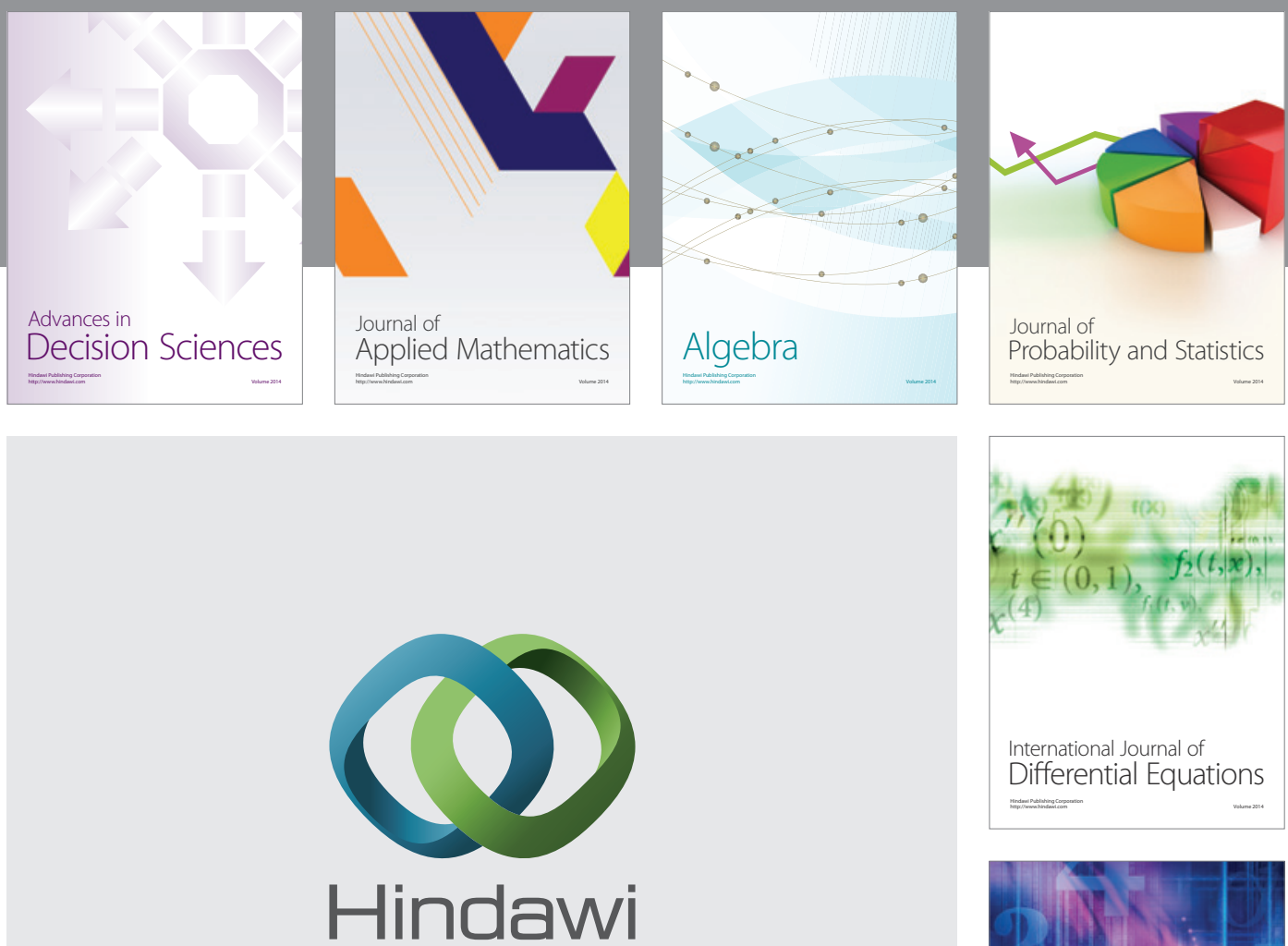

Submit your manuscripts at http://www.hindawi.com
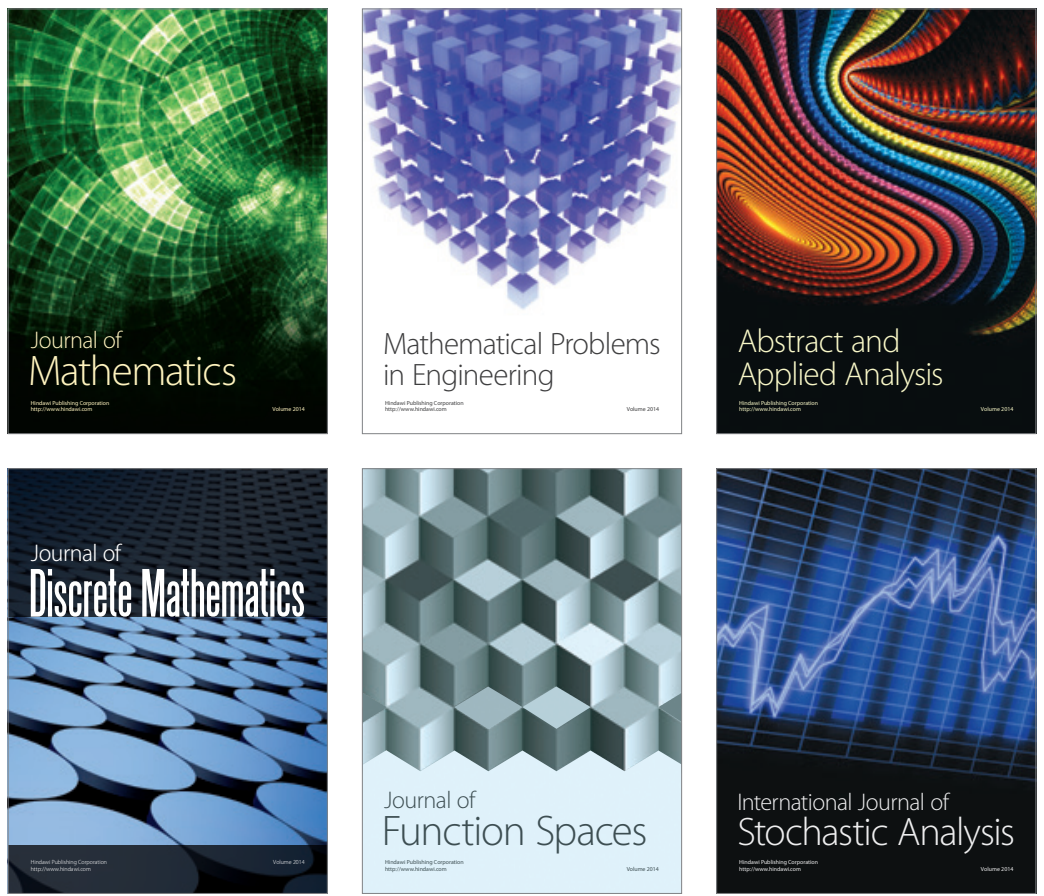

Journal of

Function Spaces

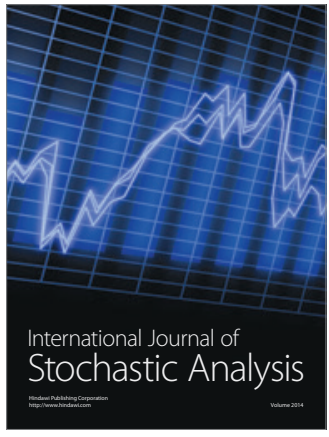

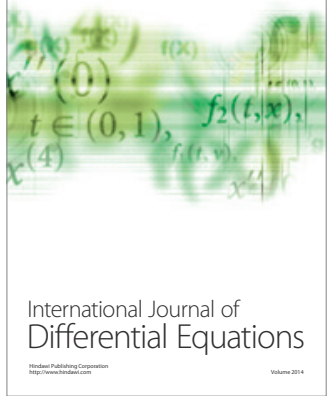
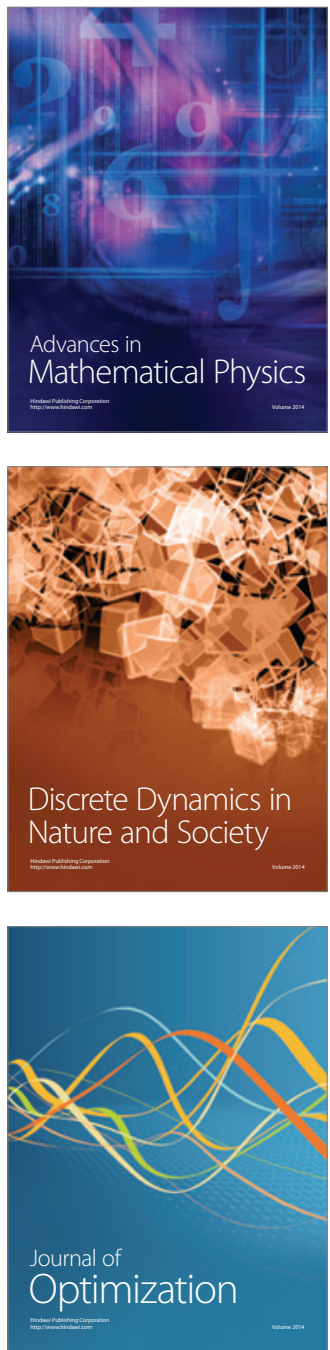